\title{
TWO VARIABLE DEFORMATIONS OF THE CHEBYSHEV MEASURE
}

\author{
JEFFREY S. GERONIMO AND PLAMEN ILIEV
}

\begin{abstract}
We construct one and two parameter deformations of the two dimensional Chebyshev polynomials with simple recurrence coefficients, following the algorithm in 3 . Using inverse scattering techniques, we compute the corresponding orthogonality measures.
\end{abstract}

\section{INTRODUCTION}

The general theory of bivariate orthogonal polynomials goes back to the work of Jackson [12. Special examples have arisen in studies related to symmetric groups [4, 16, 22]), as extensions of one variable polynomials [7, 15] and as eigenfunctions of partial differential equations [19, 14, 13, 21. An updated account of the theory can be found in the books [5, 25].

A major difficulty encountered in the theory of orthogonal polynomials of more than one variable is which monomial ordering to use. Starting with [12, the preferred ordering is the total degree ordering and for polynomials with the same total degree the ordering is lexicographical that is

$$
(k, l)<_{\text {totaldeg }}\left(k_{1}, l_{1}\right)
$$

if

$$
k+l<k_{1}+l_{1} \text { or }\left(k+l=k_{1}+l_{1}(k, l)<_{\operatorname{lex}}\left(k_{1}, l_{1}\right)\right),
$$

where lex means the lexicographical ordering (see below). In other words, we apply the Gram-Schmidt process to the polynomials ordered as follows $\left\{1, y, x, y^{2}, x y, x^{2}, \ldots\right\}$. If we denote by $\mathbf{P}_{n}(x, y)$ the $(n+1)$ dimensional vector with components the orthonormal polynomials of total degree $n$, then the multiplications by $x$ and $y$ are given by three term recurrence relations

$$
\begin{aligned}
& x \mathbf{P}_{n}=A_{x, n} \mathbf{P}_{n+1}+B_{x, n} \mathbf{P}_{n}+A_{x, n-1}^{t} \mathbf{P}_{n-1} \\
& y \mathbf{P}_{n}=A_{y, n} \mathbf{P}_{n+1}+B_{y, n} \mathbf{P}_{n}+A_{y, n-1}^{t} \mathbf{P}_{n-1},
\end{aligned}
$$

where $A_{x, n}, A_{y, n}$ are $(n+1) \times(n+2)$ matrices such that $\operatorname{rank}\left(A_{x, n}\right)=\operatorname{rank}\left(A_{y, n}\right)=$ $n+1$ and $B_{x, n}, B_{y, n}$ are symmetric $(n+1) \times(n+1)$ matrices. Notice that the Jacobi matrices corresponding to multiplications by $x$ and $y$ will commute which amounts to certain commutativity relations between the matrices defined above, see [5] for more details.

Inspired by the progress made in 10 in connection with Fejér-Riesz factorizations and autoregressive filters in two variables, an alternative way to approach two dimensional orthogonal polynomials was proposed in 3. by relating them to

Date: December 16, 2006.

JSG was partially supported by an NSF grant. 
the theory of matrix valued orthogonal polynomials. This can be accomplished by using the lexicographical ordering,

$$
(k, l)<_{\operatorname{lex}}\left(k_{1}, l_{1}\right) \Leftrightarrow k<k_{1} \text { or }\left(k=k_{1} \text { and } l<l_{1}\right),
$$

or the reverse lexicographical ordering

$$
(k, l)<_{\text {revlex }}\left(k_{1}, l_{1}\right) \Leftrightarrow(l, k)<_{\text {lex }}\left(l_{1}, k_{1}\right),
$$

to arrange the monomials. This naturally connects the theory of bivariate orthogonal polynomials to doubly Hankel matrices. In particular, this led to an alternate parametrization for positive doubly Hankel matrices and an algorithm for constructing their inverses.

In the present paper we apply the algorithm from 3 to construct deformations of the two dimensional Chebyshev polynomials with relatively simple recurrence coefficients. These polynomials can be thought of as two dimensional analogs of the Bernstein-Szegö polynomials. Using the connection of the above theory with matrix valued orthogonal polynomials and the inverse scattering techniques developed in [9] we show how one can obtain the orthogonality measure from the recurrence coefficients.

The paper is organized as follows. In the next section we recall the main ingredients needed for the construction. In particular, we review the parametrization of the doubly Hankel matrices in $[3]$ and the algorithm which gives the recurrence coefficients. We also sketch the inverse scattering techniques in the matrix case and the connection with the Darboux transformation. In Sections 3 and 4, respectively, we present one parameter and two parameter deformations of the recurrence coefficients for the two dimensional Chebyshev polynomials and we derive the orthogonality measure.

\section{Bivariate Orthogonal Polynomials}

Let $\mu(x, y)$ be a Borel measure supported on $\mathbb{R}^{2}$, such that

$$
\int_{\mathbb{R}^{2}} f(x, y) d \mu(x, y)<\infty
$$

for every polynomial $f(x, y)$. For every nonnegative integer $m$ we order the monomials $x^{i} y^{j}, 0 \leq i \leq n, 0 \leq j \leq m$ lexicographically i.e.

$$
\left\{1, y, \ldots, y^{m}, x, x y, \ldots, x y^{m}, x^{2}, \ldots\right\} .
$$

We construct the moment matrix

$$
H_{n, m}=\left[\begin{array}{cccc}
H_{0} & H_{1} & . & H_{n} \\
H_{1} & H_{2} & & H_{n+1} \\
. & & . & . \\
H_{n} & H_{n+1} & . & H_{2 n}
\end{array}\right],
$$


where each $H_{i}$ is an $(m+1) \times(m+1)$ matrix of the form

$$
H_{i}=\left[\begin{array}{cccc}
h_{i, 0} & h_{i 1} & . & h_{i, m} \\
h_{i 1} & h_{i 2} & . & \\
. & . & & . \\
h_{i, m} & & . & h_{i, 2 m}
\end{array}\right], \quad i=0, \ldots, 2 n .
$$

Thus $H_{n, m}$ is a block Hankel matrix where each block is a Hankel matrix so it has a doubly Hankel structure. If the reverse lexicographical ordering is used in place of the lexicographical ordering we obtain another moment matrix $\tilde{H}_{n, m}$ where the roles of $n$ and $m$ are interchanged. We will assume that $H_{n, m}$ is positive definite for all $n$ and $m$. For every nonnegative integer $m$ we apply the Gram-Schmidt process to the basis of monomials ordered as above and define the orthonormal polynomials $p_{n, m}^{l}(x, y), 0 \leq n, 0 \leq m, 0 \leq l \leq m$, by the equations,

$$
\begin{aligned}
& \int_{\mathbb{R}^{2}} p_{n, m}^{l} x^{i} y^{j} d \mu(x, y)=0, \quad 0 \leq i<n \text { and } 0 \leq j \leq m \text { or } i=n \text { and } 0 \leq j<l, \\
& \int_{\mathbb{R}^{2}} p_{n, m}^{l} p_{n, m}^{l} d \mu(x, y)=1,
\end{aligned}
$$

and

$$
p_{n, m}^{l}(x, y)=k_{n, m, l}^{n, l} x^{n} y^{l}+\sum_{(i, j)<\operatorname{lex}(n, l)} k_{n, m, l}^{i, j} x^{i} y^{j} .
$$

With the convention $k_{n, m, l}^{n, l}>0$, the above equations uniquely specify $p_{n, m}^{l}$. Polynomials orthonormal with respect to $d \mu(x, y)$ but using the reverse lexicographical ordering will be denoted by $\tilde{p}_{n, m}^{l}$. They are uniquely determined by the above relations with the roles of $n$ and $m$ interchanged. Set,

$$
\mathbb{P}_{n, m}=\left[\begin{array}{c}
p_{n, m}^{0} \\
p_{n, m}^{1} \\
\vdots \\
p_{n, m}^{m}
\end{array}\right] .
$$

The $\mathbb{P}_{n, m}$ may be obtained in an alternate manner as follows. We associate an $(m+1) \times(m+1)$ matrix valued measure $d M^{m+1}(x)$ by taking

$$
d M^{m+1}(x)=\int_{\mathbb{R}}\left[1, y, \ldots, y^{m}\right]^{t} d \mu_{y}(x, y)\left[1, y, \ldots, y^{m}\right],
$$

where the above integral is with respect to $y$. Let us denote by $\left\{P_{n, m}\right\}_{n=0}^{\infty}$ the sequence of $(m+1) \times(m+1)$ matrix valued polynomials satisfying

$$
\begin{array}{r}
P_{n, m}(x)=K_{n, n}^{m} x^{n}+\text { lower order terms, } \\
\int_{\mathbb{R}} P_{n, m}(x) d M^{m+1}\left(P_{k, m}(x)\right)^{t}=\delta_{k, m} I_{m+1},
\end{array}
$$

with $K_{n, n}^{m}$ a lower triangular matrix with strictly positive diagonal entries. The above conditions uniquely specify these left matrix valued orthogonal polynomials and it follows that

$$
\mathbb{P}_{n, m}(x, y)=P_{n, m}(x)\left[1, y, \ldots, y^{m}\right]^{t} .
$$


From equation (2.6) we see that $M^{m+1}$ is a Hankel matrix and we have,

Theorem 2.1. Let $M^{m+1}(x)$ be a matrix measure supported on some interval $I \subset \mathbb{R}$ then $M^{m+1}(x)$ is a Hankel measure if and only if there exists a bounded positive linear functional $\mathcal{F}_{2 m}: C(I) \times\left\{1, \ldots, y^{2 m}\right\} \rightarrow \mathbb{R}$ with $\left(\int_{I} x^{k} d M^{m+1}\right)_{i, j}=$ $\mathcal{F}_{2 m}\left(x^{k} y^{i+j}\right)$.

Proof. If we begin with $M^{m+1}(x)$ set $H_{i}=\int_{I} x^{i} d M^{m+1}$ and let $H_{n, m}$ be the $(n+1)(m+1) \times(n+1)(m+1)$ matrix given by equation (2.1). Then $H_{n, m}$ is a positive definite doubly Hankel matrix which implies via the above formula connecting $M^{m+1}$ and $\mathcal{F}_{2 m}$ and the density of the polynomials in $C(I)$ that $\mathcal{F}_{2 m}$ is a bounded positive linear functional which is uniquely defined. The converse follows from the relation between $\mathcal{F}_{2 m}$ and $M^{m+1}$ given above.

The following Theorem was proved in [3],

Theorem 2.2. Given $\left\{\mathbb{P}_{n, m}\right\}$ and $\left\{\tilde{\mathbb{P}}_{n, m}\right\}$ the following recurrence formulas hold,

$$
\begin{aligned}
& x \mathbb{P}_{n, m}=A_{n+1, m} \mathbb{P}_{n+1, m}+B_{n, m} \mathbb{P}_{n, m}+A_{n, m}^{\top} \mathbb{P}_{n-1, m}, \\
& \Gamma_{n, m} \mathbb{P}_{n, m}=\mathbb{P}_{n, m-1}-\mathcal{K}_{n, m} \tilde{\mathbb{P}}_{n-1, m}, \\
& J_{n, m}^{1} \mathbb{P}_{n, m}=y \mathbb{P}_{n, m-1}+J_{n, m}^{2} \tilde{\mathbb{P}}_{n-1, m}+J_{n, m}^{3} \tilde{\mathbb{P}}_{n-1, m-1}, \\
& \mathbb{P}_{n, m}=I_{n, m} \tilde{\mathbb{P}}_{n, m}+\Gamma_{n, m}^{\top} \mathbb{P}_{n, m-1}
\end{aligned}
$$

with

$$
\begin{aligned}
A_{n, m} & =\left\langle x \mathbb{P}_{n-1, m}, \mathbb{P}_{n, m}\right\rangle, \\
B_{n, m} & =\left\langle x \mathbb{P}_{n, m}, \mathbb{P}_{n, m}\right\rangle \\
J_{n, m}^{1} & =\left\langle y \mathbb{P}_{n, m-1}, \mathbb{P}_{n, m}\right\rangle \\
J_{n, m}^{2} & =-\left\langle y \mathbb{P}_{n, m-1}, \tilde{\mathbb{P}}_{n-1, m}\right\rangle \\
J_{n, m}^{3} & =-\left\langle y \mathbb{P}_{n, m-1}, \tilde{\mathbb{P}}_{n-1, m-1}\right\rangle \\
\Gamma_{n, m} & =\left\langle\mathbb{P}_{n, m-1}, \mathbb{P}_{n, m}\right\rangle \\
\mathcal{K}_{n, m} & =\left\langle\mathbb{P}_{n, m-1}, \tilde{\mathbb{P}}_{n-1, m}\right\rangle \\
I_{n, m} & =\left\langle\mathbb{P}_{n, m}, \tilde{\mathbb{P}}_{n, m}\right\rangle
\end{aligned}
$$

where for every two vector valued polynomials $f, g$ we set

$$
\langle f, g\rangle=\int_{\mathbb{R}^{2}} f(x, y) g^{t}(x, y) d \mu(x, y) .
$$

Similar formulas hold for $\tilde{\mathbb{P}}_{n, m}(x, y)$.

Equation (2.10) comes from the fact that the vector polynomials can be related to matrix orthogonal polynomials as discussed above. Given the coefficients in this equation it is possible to compute the vector polynomials in a strip of size $m+1$ in the $n$ direction. Its tilde counterpart obtained from the reverse lexicographical ordering allows one to compute the tilde vector polynomials along a strip of size $n+1$ in the $m$ direction. Equations (2.11) and (2.12) allow one to compute by changing $n$ and $m$ simultaneously. That is given the polynomials at the $(n, m-1)$ level and the $(n-1, m)$ level and the coefficients in (2.11), (2.12) and (2.13) it is possible to compute $\mathbb{P}_{n, m}$ and $\tilde{\mathbb{P}}_{n, m}$. It should be noted that in going from $(n-1, m)$, and $(n, m-1)$ to $(n, m)$ there are generically 4 new moments. Examination of the 
sizes of the coefficients in (2.11) and (2.12) shows that most of the coefficients in these matrices must be computable from the coefficients given at previous levels. Indeed in [3] it was shown that this is the case and that for $n \geq 1$ and $m \geq 1$ only $\left(\mathcal{K}_{n, m}\right)_{m, n},\left(J_{n, m}^{2}\right)_{m, n},\left(J_{n, m}^{1}\right)_{m, m}$ and $\left(J_{n, m}^{1}\right)_{m, m+1}$ need be specified in order to compute $\mathbb{P}_{n, m}$. For $n \geq 1$ and $m \geq 1$ we denote

$$
\begin{gathered}
\left(\mathcal{K}_{n, m}\right)_{m, n}=s_{2 n-1,2 m-1} \quad\left(J_{n, m}^{2}\right)_{m, n}=s_{2 n-1,2 m} \\
\left(J_{n, m}^{1}\right)_{m, m}=s_{2 n, 2 m-1} \quad\left(J_{n, m}^{1}\right)_{m, m+1}=s_{2 n, 2 m} .
\end{gathered}
$$

For $n=0$ and $m \geq 0$ or $n \geq 1$ and $m=0$ we introduce $s_{0, m}$ and $s_{n, 0}$ which are parameters in the one dimensional recurrence formulas associated with the line $(n, 0)$ or $(0, m)$ respectively. Thus we find

Theorem 2.3. 3] Given parameters $s_{0,0}, \ldots, s_{2 n, 2 m} \in \mathbb{R}$, we construct

- scalars $A_{i+1,0}, B_{i, 0}, i=0, \ldots, n-1$, and $\tilde{A}_{0, j+1}, \tilde{B}_{0, j}, j=0, \ldots, m-1$;

- $j \times i$ matrices $\mathcal{K}_{i, j}$ and $J_{i, j}^{2}, i=1, \ldots, n, j=1, \ldots, m$;

- $j \times(j+1)$ matrices $J_{i, j}^{1}$ for $i=1, \ldots, n, j=1,2 \ldots, m$.

If

$$
s_{2 i, 2 j}>0 \text { and }\left\|\mathcal{K}_{i, j}\right\|<1,
$$

then there exists a positive linear functional $\mathcal{F}$ such that

$$
\mathcal{F}\left(\mathbb{P}_{i, m}, \mathbb{P}_{j, m}\right)=\delta_{i, j} I_{m+1} \text { and } \mathcal{F}\left(\tilde{\mathbb{P}}_{n, i}, \tilde{\mathbb{P}}_{n, j}\right)=\delta_{i, j} I_{n+1} .
$$

The conditions (2.17) are also necessary.

With the above we are able to begin with the parameters $s_{i, j}$ and try to compute two variable orthogonality measures associated with these parameters. The connection with matrix orthogonal polynomials will be especially useful and we review some of the relevant relations. The theory of matrix orthogonal polynomials has an extensive literature [1, 6, 9, 23, 24, 26] and we begin with the equation (2.10) and use (2.9) to find,

$$
x P_{n, m}=A_{n+1, m} P_{n+1, m}+B_{n, m} P_{n, m}+A_{n, m}^{t} P_{n-1, m} .
$$

If we set $P_{-1, m}=0$ and $P_{0, m}=I_{m+1}$ then the above equation uniquely gives $\left\{P_{n, m}\right\}$. We now turn the problem around and begin with $\left\{A_{n, m}\right\}_{n=1}^{\infty}$ and $\left\{B_{n, m}\right\}_{n=0}^{\infty}$ where $A_{i, m} B_{i-1, m}$ are $(m+1) \times(m+1)$ real valued matrices with $B_{n, m}=B_{n, m}^{t}$ and $A_{n, m}$ lower triangular with strictly positive diagonal entries. We will suppose that

$$
\lim _{n \rightarrow \infty} A_{n, m}=\frac{1}{2} I_{m+1} \text { and } \lim _{n \rightarrow \infty} B_{n, m}=0,
$$

and define $A_{0, m}=I_{m+1}$. In this case, there is a unique matrix measure $M$ such that

$$
\int_{-1}^{1} P_{n, m}(x) d M(x) P_{k, m}(x)^{t}=\delta_{n, k} I_{m+1} .
$$

Following [9] we introduce the matrix valued function

$$
\Psi_{n, m}(z)=P_{n, m}-2 z A_{n, m}^{t} P_{n-1, m},
$$

where $z=x-\sqrt{x^{2}-1}$. The branch of the square root is chosen so that $z \rightarrow 0$ as $x \rightarrow+\infty$. Using (2.19) and (2.22) one can deduce that

$$
\begin{aligned}
& \Psi_{n, m}(z) \\
& =\frac{1}{2 z} A_{n, m}^{-1} \Psi_{n-1, m}+\frac{1}{2} A_{n, m}^{-1}\left[\left(I_{m+1}-4 A_{n, m} A_{n, m}^{t}\right) z-2 B_{n-1, m}\right] P_{n-1, m} .
\end{aligned}
$$


Note that if $A_{n, m}=\frac{1}{2} I_{m+1}$ and $B_{n-1, m}=0$ for all $n \geq n_{0}$ then

$$
\Psi_{n, m}^{*}(z)=z^{n} \Psi_{n, m}(z)=\Psi_{n_{0}, m}^{*}(z) \text { for all } n \geq n_{0} .
$$

We also introduce two scattering solutions of (2.19) $P_{n, m}^{ \pm}(z)$ that satisfy

$$
\lim _{n \rightarrow \infty}\left\|z^{\mp n} P_{n, m}^{ \pm}(z)-I_{m+1}\right\|=0
$$

where the matrix norm is the Hilbert-Schmidt norm i.e $\|B\|=\left(\operatorname{tr}\left(B B^{\dagger}\right)\right)^{1 / 2}$. If we assume

$$
\sum_{n=1}^{\infty} n\left(\left\|1-4 A_{n, m} A_{n, m}^{t}\right\|+\left\|B_{n-1, m}\right\|\right)<\infty,
$$

then a slight modification of the techniques in 9] gives that for $n \geq 0, P_{n, m}^{+}$exists, is continuous for $|z| \leq 1$ and analytic for $|z|<1$ while $P_{n, m}^{-}$exists, is continuous for $|z| \geq 1$ and analytic for $|z|>1$. Since the coefficients in (2.19) are real valued we see from the asymptotic conditions satisfied by $P_{n, m}^{ \pm}$that for $|z|=1, P_{n, m}^{-}(z)=$ $P_{n, m}^{+}(1 / z)$. For two solutions $Y$ and $X$ of (2.19) we define

$$
W_{m}[X, Y]=X_{n, m}^{\dagger}(\bar{x}) A_{n+1, m} Y_{n+1, m}(x)-X_{n+1, m}^{\dagger}(\bar{x}) A_{n+1, m}^{t} Y_{n, m}(x),
$$

which by standard computations is independent of $n$. With the use of $W_{m}$ it follows that if (2.24) holds then for all $|z|=1, z \neq \pm 1$

$$
P_{n, m}(x)=\frac{2}{z-1 / z}\left(P_{n, m}^{+}(z) f_{-}(z)-P_{n, m}^{-}(z) f_{+}(z)\right)
$$

where

$$
f_{+}(z)=P_{-1, m}^{+}(z)^{t} \quad|z| \leq 1
$$

and

$$
f_{+}(z)=\frac{1}{2 z} \lim _{n \rightarrow \infty} \Psi_{n, m}^{*}(z),|z| \leq 1 .
$$

From the relation between $P_{n, m}^{ \pm}$we find,

$$
f_{+}(z)=f_{-}(1 / z) \quad|z|=1
$$

Manipulations similar to those that lead to (2.25) yield

$$
\begin{aligned}
& \left(P_{n, m}^{+}(z)\right)^{\dagger} A_{n+1, m} P_{n+1, m}^{+}(z)-\left(P_{n+1, m}^{+}\right)^{\dagger}(z) A_{n+1, m}^{t} P_{n, m}^{+}(z) \\
& =\frac{1}{2}(z-1 / z) I_{m+1}, \quad|z|=1, \quad z \neq \pm 1
\end{aligned}
$$

which implies that $P^{+}$is nonsingular for $|z|=1, z \neq \pm 1$. This follows since if there is a vector a and a $z_{0},\left|z_{0}\right|=1, z_{0} \neq \pm 1$ such that $P_{n, m}^{+}\left(z_{0}\right) \mathbf{a}=0$ then $\left(P_{n, m}^{+}\left(z_{0}\right) \mathbf{a}\right)^{\dagger}=0$ which cannot happen by the equation above. This implies that $f_{+}$ is nonsingular for $|z|=1, z \neq \pm 1$. With $n=-1$ in (2.26) we find for $|z|=1, z \neq \pm 1$ the useful equation

$$
f_{+}^{t} f_{-}=f_{-}^{t} f_{+} .
$$

Another useful relation between $f_{+}$and $P_{n, m}^{+}$is [9, formula 7.3],

$$
P_{n, m}^{+}(z)=\int_{-\infty}^{\infty} \frac{P_{n, m}(y)}{x-y} d M(y) f_{+}^{t}(z) \quad|z|<1
$$

where the relation between $x$ and $z$ is given above.

We will now make the following assumptions

$$
A_{n+1, m}=\frac{1}{2} I_{m+1}, \quad B_{n, m}=0, \forall n \geq n_{0},
$$


and

$$
\operatorname{det}\left(z f_{+}(z)\right) \neq 0, \quad|z| \leq 1,
$$

which will simplify the presentation below and are sufficiently general for our examples. The more general case will be taken up later (see however 9] and 24]). With assumption (2.33) we see from (2.28) and (2.23) that $2 z f_{+}(z)=\Psi_{n_{0}, m}^{*}(z)$ which will be a matrix polynomial of degree $2 n_{0}$ if $A_{n_{0}, m} \neq \frac{1}{2} I_{m+1}$ and of degree $2 n_{0}-1$ if $A_{n_{0}, m}=\frac{1}{2} I_{m+1}$ and $B_{n_{0}-1, m} \neq 0$ and for $n \geq 0, P_{n, m}^{ \pm}$are matrix polynomials in $z^{ \pm 1}$ respectively. Furthermore (2.26) and contour integration show that the matrix orthogonal polynomials $\left\{P_{n, m}\right\}$ satisfy equation (2.21) with

$$
d M(x)=\sigma_{m}(x) d x=\frac{1}{2 \pi} \sqrt{1-x^{2}}\left(f_{+}(z)^{\dagger} f_{+}(z)\right)^{-1} d x, \quad z=e^{i \theta} .
$$

What we would like to calculate next is what happens when we add a mass point to the system which can be accomplished by multiplying $\Psi_{n_{0}, m}$ by a zero. To this end write,

$$
\hat{\Psi}_{n_{0}+1, m}(z)=\left(z_{0} / z-1\right) \Psi_{n_{0}, m}(z),
$$

where $z_{0}$ is real and $\left|z_{0}\right|<1$. With the choice of $\hat{\Psi}_{n_{0}+1, m}$ the computation of the orthogonality measure as well as the matrix orthogonal polynomials $\left\{\hat{P}_{n, m}(x)\right\}$ with $\hat{P}_{0, m}=I_{m+1}$ can be carried out in the following manner,

Theorem 2.4. Given $\left\{A_{n, m}\right\}$ and $\left\{B_{n, m}\right\}$ satisfying (2.33) and $\Psi_{n_{0}, m}$ satisfying (2.34). Let $\hat{\Psi}_{n_{0}+1, m}$ be given by (2.36). Then $\left\{\hat{P}_{n, m}(x)\right\}$ are a set of matrix orthogonal polynomials satisfying

$$
\int_{\mathbb{R}} \hat{P}_{n, m}(x) d \hat{M}(x) \hat{P}_{k, m}(x)^{t} d x=I_{m+1} \delta_{n, k} \quad n, k \geq 0
$$

where

$$
d \hat{M}(x)=\left\{\begin{array}{l}
\frac{\hat{\sigma}_{m}(x)}{x_{0}-x}, \quad-1<x<1 \\
\hat{r}_{m} \delta\left(x-x_{0}\right), \quad x \notin[-1,1]
\end{array}\right.
$$

with

$$
\hat{\sigma}_{m}(x)=\frac{\sigma_{m}(x)}{2 z_{0}}
$$

and

$$
\hat{r}_{m}=-\frac{\left(z_{0}-1 / z_{0}\right)}{4 z_{0}}\left[f_{+}\left(1 / \bar{z}_{0}\right)^{\dagger} f_{+}\left(z_{0}\right)\right]^{-1} .
$$

The polynomials $\left\{\hat{P}_{n, m}\right\}$ have the Uvarov-Christoffel representation,

$$
\hat{P}_{n, m}(x)=d_{n, m}\left(Q_{n-1, m}\left(x_{0}\right)^{\dagger} A_{n, m} P_{n, m}(x)-Q_{n, m}\left(x_{0}\right)^{\dagger} A_{n, m}^{t} P_{n-1, m}(x)\right),
$$

where

$$
Q_{n, m}\left(x_{0}\right)=\int_{-\infty}^{\infty} P_{n, m}(x) d \hat{M}(x) .
$$

The constants $d_{n, m}$ are chosen so that $\hat{P}_{n, m}$ is orthonormal for $n \geq 0$, which is equivalent to

$$
\left(d_{n, m}^{t} d_{n, m}\right)^{-1}=\frac{1}{\left(2 z_{0}\right)^{3}} f_{-}\left(z_{0}\right)^{-1} P_{n-1, m}^{-}\left(z_{0}\right)^{t} A_{n, m} P_{n, m}^{-}\left(z_{0}\right) f_{-}^{t}\left(z_{0}\right)^{-1} .
$$


Proof. From the definition of $Q_{n, m}(x), d \hat{M}(x)$ and $\hat{\sigma}_{m}$ we find for $n>0$ and $0<i<n$ that

$$
\int_{-\infty}^{\infty} \hat{P}_{n, m}(x)\left(x-x_{0}\right)^{i} d \hat{M}(x)=0 .
$$

Equations (2.32), (2.31) and (2.26) show that

$$
Q_{n}\left(x_{0}\right)=P_{n, m}^{+}\left(z_{0}\right) f_{+}^{t}\left(z_{0}\right)^{-1} / 2 z_{0}+P_{n, m}\left(x_{0}\right) \hat{r}_{m}=P_{n, m}^{-}\left(z_{0}\right) f_{-}^{t}\left(z_{0}\right)^{-1} / 2 z_{0},
$$

where we have used the definition of $\hat{r}_{m}$ and the analytic properties of $P_{n, m}^{ \pm}$. We use the above equation to define $Q_{-1, m}$. The substitution of this formula into (2.44) with $i=0$ yields

$$
\begin{aligned}
& d_{n, m}^{-1} \int_{-\infty}^{\infty} \hat{P}_{n, m}(x) d \hat{M}(x) \\
& =\frac{1}{\left(2 z_{0}\right)^{2}}\left(f_{-}\left(z_{0}\right)^{-1}\left(P_{n-1, m}^{-}\left(z_{0}\right)^{t} A_{n, m} P_{n, m}^{+}\left(z_{0}\right)-P_{n, m}^{-}\left(z_{0}\right)^{t} A_{n, m}^{t} P_{n-1, m}^{+}\left(z_{0}\right)\right) f_{+}^{t}\left(z_{0}\right)^{-1}\right. \\
& +\frac{1}{2 z_{0}}\left(f_{-}\left(z_{0}\right)^{-1}\left(P_{n-1, m}^{-}\left(z_{0}\right)^{t} A_{n, m} P_{n, m}\left(x_{0}\right)-P_{n, m}^{-}\left(z_{0}\right)^{t} A_{n, m}^{t} P_{n-1, m}\left(x_{0}\right)\right) \hat{r}_{m} .\right.
\end{aligned}
$$

If in the first term on the left hand side we use (2.25) and let $n$ tend to $\infty$ while in the second term we use (2.25) and set $n=0$ we find from the definition of $\hat{r}_{m}$ and $f_{+}$that the above integral is equal to 0 for $n>0$. To show that $d_{n, m}$ is given as in equation (2.43) we begin with the recurrence formula satisfied by $Q_{n, m}\left(x_{0}\right)$

$$
x_{0} Q_{n, m}=A_{n+1, m} Q_{n+1, m}+B_{n, m} Q_{n, m}+A_{n, m}^{t} Q_{n-1, m},
$$

which follows from (2.42). Thus routine manipulations give

$$
d_{n, m}^{-1} \hat{P}_{n, m}(x)=\left(x-x_{0}\right) \sum_{i=0}^{n-1} Q_{i, m}^{\dagger}\left(x_{0}\right) P_{i, m}(x)+Q_{-1, m}\left(x_{0}\right),
$$

so that for $n>0$

$$
\begin{aligned}
& \int_{\mathbb{R}} \hat{P}_{n, m}(x) d \hat{M}(x) \hat{P}_{n, m}^{t}(x) \\
& =-\frac{1}{2 z_{0}} d_{n, m} \sum_{i=0}^{n-1} Q_{i, m}^{\dagger}\left(z_{0}\right) \int_{-1}^{1} P_{i, m}(x) d M(x) \hat{P}_{n, m}^{t}(x) \\
& =\frac{1}{2 z_{0}} d_{n, m} Q_{n-1, m}^{t}\left(z_{0}\right) A_{n, m} Q_{n, m}\left(z_{0}\right) d_{n, m}^{t} .
\end{aligned}
$$

The last equality follows by eliminating $\hat{P}_{n, m}$ using its definition.

Remark 2.5. If we want to fix $d_{n, m}$ so that the highest coefficient of $\hat{P}_{n, m}(x)$ is a lower triangular matrix, we need to choose in (2.43) the unique solution for which $d_{n, m} Q_{n-1, m}\left(x_{0}\right)^{\dagger}$ is a lower triangular matrix. Notice that for $n \geq n_{0}$ we have $P_{n, m}^{-}\left(z_{0}\right)=z_{0}^{-n} I_{m+1}, A_{n+1, m}=\frac{1}{2} I_{m+1}$ which leads to $d_{n+1, m}=4 z_{0}^{n+2} f_{-}\left(z_{0}\right)$. Thus for $n>n_{0}$, formula (2.41) reduces to $\hat{P}_{n, m}=z_{0} P_{n, m}-P_{n-1, m}$. This implies $\hat{A}_{n+1, m}=\frac{1}{2} I_{m+1}$ and $\hat{B}_{n, m}=0$ for $n>n_{0}$.

Remark 2.6. Theorem 2.4 can be easily explained in terms of the Darboux transformation. Indeed, let $\mathcal{L}_{m}$ be the second order difference operator corresponding to the second order difference equation (2.19) for the polynomials $P_{n, m}(x)$, i.e. 
$\left(\mathcal{L}_{m}(f)\right)_{n}=A_{n+1, m} f_{n+1}+B_{n, m} f_{n}+A_{n, m}^{t} f_{n-1}$. We can think of $\mathcal{L}_{m}$ as a second order difference operator acting on matrix valued functions (the size of the matrices is $(m+1) \times(m+1))$ of a discrete variable $n$. Similarly, we denote by $\hat{\mathcal{L}}_{m}$ the operator corresponding to the polynomials $\hat{P}_{n, m}(x)$. Then using (2.47) one can check that operator $\mathcal{L}_{m}-x_{0} \mathrm{Id}$ can be factored as

$$
\mathcal{L}_{m}-x_{0} \mathrm{Id}=\mathcal{P} Q
$$

where $Q$ is the backward difference operator in (2.41), i.e.

$$
\mathcal{Q}(f)_{n}=d_{n, m}\left(Q_{n-1, m}\left(x_{0}\right)^{\dagger} A_{n, m} f_{n}-Q_{n, m}\left(x_{0}\right)^{\dagger} A_{n, m}^{t} f_{n-1}\right)
$$

and $\mathcal{P}$ is the forward difference operator

$$
\mathcal{P}(f)_{n}=\left(Q_{n, m}^{\dagger}\right)^{-1}\left(f_{n+1} d_{n+1, m}^{-1}-f_{n} d_{n, m}^{-1}\right) .
$$

The operator $\hat{\mathcal{L}}_{m}$ is obtained from $\mathcal{L}_{m}$ by exchanging the factors in (2.49), i.e.

$$
\hat{\mathcal{L}}_{m}-x_{0} \mathrm{Id}=\mathrm{QP} .
$$

The above discussion can be summarized as,

Theorem 2.7. Suppose that the coefficients in $\mathcal{L}_{m}$ satisfy $(2.33)$ and $z f_{+}(z)$ satisfies (2.34). If $\hat{\mathcal{L}}_{m}$ is related to $\mathcal{L}_{m}$ by the Darboux transformation (2.49) and (2.51) and $Q$ is a backward difference operator of the form (2.50) where $Q_{n, m}\left(x_{0}\right)$ are given by (2.45) then the polynomials $\hat{P}_{n, m}$ are orthogonal with respect to $\hat{M}$ which is given by equations (2.37) -(2.40).

We can now use the above results to help solve the bivariate problem via the parametric moment problem. Equation (2.6) and Theorem[2.1] show that $d M^{m+1}$ is a Hankel matrix with entries $d \mu_{j}(x)=\int y^{j} d \mu(x, y)$ with the integration being over $y$. The above construction allows us to compute $\mu_{j}(x)$ for every $j$. We can think of $\mu_{j}(x)$ as one dimensional moments (in the variable $y$ ), depending on a parameter $x$. Let us consider the corresponding polynomials $q_{m}^{x}(y)$. The three term recurrence formula takes the form

$$
y q_{m}^{x}(y)=a_{m+1}^{x} q_{m+1}^{x}(y)+b_{m}^{x} q_{m}^{x}(y)+a_{m}^{x} q_{m-1}^{x}(y),
$$

with coefficients depending also on the parameter $x$. Next we use one dimensional theory and compute $d \mu(x, y)$ by introducing the function

$$
\psi_{m}^{x}(w)=q_{m}^{x}-2 w a_{m}^{x} q_{m-1}^{x}
$$

We will return to this general strategy in a later paper and will be content to illustrate it with the examples in the next section.

\section{One parameter deformation of the Chebyshev polynomials}

The two dimensional Chebyshev polynomials corresponding to the measure

$$
\frac{4}{\pi^{2}} \sqrt{1-x^{2}} \sqrt{1-y^{2}} d x d y
$$

are parametrized in terms of the $s_{i, j}$ from Theorem 2.3 as follows $s_{0,0}=1, s_{2 n-1,0}=$ $0, s_{2 n, 0}=\frac{1}{2}, s_{0,2 m-1}=0, s_{0,2 m}=\frac{1}{2}, s_{2 n-1,2 m-1}=0, s_{2 n-1,2 m}=0, s_{2 n, 2 m-1}=0$, $s_{2 n, 2 m}=\frac{1}{2}$ for $n, m \in \mathbb{N}$.

In order to have a nontrivial two dimensional polynomials (i.e. the measure is not just a product of two one dimensional measures) it was shown in $[3]$ that $\mathcal{K}_{i, j} \neq 0$ for at least one pair of indexes $(i, j) \in \mathbb{N}^{2}$. One way to construct such polynomials 
with relatively simple recurrence coefficients is as follows. We take $\mathcal{K}_{1,1}=\left[s_{1,1}\right]$ to be a nonzero matrix, i.e. pick $s_{1,1} \neq 0$. Following the algorithm in Section 6 of [3], we pick at each level $(n, m)$ with $n, m \in \mathbb{N}$ parameters $s_{2 n-1,2 m-1}=s_{1,1}$, $s_{2 n-1,2 m}=0, s_{2 n, 2 m-1}=0, s_{2 n, 2 m}=\frac{1}{2}$.

This specific choice leads to a one parameter deformation of the Chebyshev polynomials with recurrence coefficients given by the following formulas

$$
\mathcal{K}_{n, m}=\left[\begin{array}{ccccc}
0 & 0 & \cdots & 0 & 0 \\
\vdots & \vdots & & \vdots & 0 \\
0 & 0 & \cdots & 0 & 0 \\
0 & 0 & \cdots & 0 & s_{1,1}
\end{array}\right], \quad J_{n, m}^{1}=\frac{1}{2}\left[\begin{array}{cccccc}
0 & 1 & & & & \\
1 & 0 & 1 & & & \\
& & \ddots & \ddots & & \\
& & 1 & 0 & 1 \\
& & & \sqrt{1-s_{1,1}^{2}} & 0 & 1
\end{array}\right],
$$

and $J_{n, m}^{2}$ is a zero matrix. For $A_{n, m}$ and $B_{n, m}$ we obtain

$$
\begin{gathered}
A_{1, m}=\frac{\sqrt{1-s_{1,1}^{2}}}{2}\left[\begin{array}{cccc}
1 & & & \\
& \ddots & & \\
& & & 1 \\
& & & \frac{1}{\sqrt{1-s_{1,1}^{2}}}
\end{array}\right], \\
B_{0, m}=\frac{s_{1,1}}{2}\left[\begin{array}{cccccc}
0 & 1 & & & \\
1 & 0 & 1 & & \\
& & \ddots & \ddots & & \\
& & & 1 & 0 & 1 \\
& & & & 1 & 0
\end{array}\right]
\end{gathered}
$$

and for $n \geq 2$ we have

$$
A_{n, m}=\frac{1}{2} I_{m+1}, \quad B_{n-1, m}=0,
$$

i.e. beyond $n=1$ the matrices $A_{n, m}$ and $B_{n-1, m}$ reach their asymptotic values. Similar formulas hold if we reverse the roles of $x$ and $y$, i.e. we have a measure symmetric in $x$ and $y$.

Equation (3.1) shows that the right-hand side of formula (2.23) will vanish for $n \geq 2$, which gives that

$$
\Psi_{n, m}^{*}(z)=\Psi_{1, m}^{*}(z) \text { for } n \geq 1
$$

and $f_{+}(z)=\frac{1}{2 z} \Psi_{1, m}^{*}$ by (2.28). This gives the matrix measure $d M(x)$ via formula (2.35).

The entries of the Hankel matrix $d M^{m+1}(x)$ allow us to compute the moments $\mu_{j}(x) d x=\int y^{j} d \mu_{y}(x, y)$, thus the coefficients in the three term recurrence relation (2.52).

For the first few values one obtains that

$$
a_{1}^{x}=\frac{\sqrt{1-s_{1,1}^{2}}}{2}, \quad b_{0}^{x}=s_{1,1} x
$$

and

$$
a_{m}^{x}=\frac{1}{2}, \quad b_{m-1}^{x}=0, \text { for } m \geq 2
$$


This suggest one possible way to compute the measure $d \mu(x, y)$, by first proving that (3.2) holds and then using one dimensional scattering theory in the variable $y$ with $x$ as a parameter. This would give that $w^{m} \psi_{m}^{x}(w)$ is independent of $m$ for $m \geq 1$, where $\psi_{m}^{x}(w)$ is the function defined by (2.53), i.e.

$$
w^{m} \psi_{m}^{x}(w)=w \psi_{1}^{x}(w)=\frac{s_{1,1}^{2} w^{2}-2 s_{1,1} x w+1}{\sqrt{1-s_{1,1}^{2}}} .
$$

Applying the one dimensional scattering techniques, we can show that the measure is given by the following formula

$$
\begin{aligned}
d \mu(x, y) & =\frac{4}{\pi^{2}} \frac{\sqrt{1-x^{2}} \sqrt{1-y^{2}}}{\psi_{m}^{x}(w) \overline{\psi_{m}^{x}(w)}} d x d y \\
& =\frac{4}{\pi^{2}} \frac{\left(1-s_{1,1}^{2}\right) \sqrt{1-x^{2}} \sqrt{1-y^{2}}}{4 s_{1,1}^{2}\left(x^{2}+y^{2}\right)-4 s_{1,1}\left(1+s_{1,1}^{2}\right) x y+\left(1-s_{1,1}^{2}\right)^{2}} d x d y .
\end{aligned}
$$

In our particular case, we can give a simpler proof using the connection of the polynomials above with the Chebyshev polynomials. This is the content of the next theorem.

Theorem 3.1. The polynomials $\mathbb{P}_{n, m}(x, y)$ constructed with the parameters $s_{i, j}$ given by

$$
\begin{aligned}
& s_{0,0}=1, \quad s_{2 n-1,0}=0, \quad s_{2 n, 0}=\frac{1}{2}, \quad s_{0,2 m-1}=0, \quad s_{0,2 m}=\frac{1}{2} \\
& s_{2 n-1,2 m-1}=s_{1,1}, \quad s_{2 n-1,2 m}=0, \quad s_{2 n, 2 m-1}=0, \quad s_{2 n, 2 m}=\frac{1}{2},
\end{aligned}
$$

for all $m, n \in \mathbb{N}$ are orthonormal with respect to the measure $d \mu(x, y)$ defined by (3.3).

Proof. We have

$$
\mathbb{P}_{n, m}(x, y)=P_{n, m}(x)\left[\begin{array}{c}
1 \\
y \\
\vdots \\
y^{m}
\end{array}\right]
$$

Thus the orthogonality relation

$$
\int \mathbb{P}_{n, m}(x, y) \mathbb{P}_{k, m}^{t}(x, y) d \mu(x, y)=\delta_{n, k} I_{m+1}
$$

is equivalent to

$$
\int_{-1}^{1}\left(P_{n, m}(x) \int_{-1}^{1}\left[\begin{array}{c}
1 \\
y \\
\vdots \\
y^{m}
\end{array}\right]\left[\begin{array}{llll}
1 & y & \ldots & y^{m}
\end{array}\right] d_{y} \mu\right) P_{k, m}(x)^{t} \frac{2 \sqrt{1-x^{2}}}{\pi} d x=\delta_{n, k} I_{m+1}
$$

where

$$
d_{y} \mu=\frac{\pi}{2 \sqrt{1-x^{2}}} d \mu
$$


is a positive measure in the variable $y$. From (2.35) we know that

$$
\int_{-1}^{1} P_{n, m}(x)\left(\Psi_{1}^{m}(z)^{\dagger} \Psi_{1}^{m}(z)\right)^{-1} P_{k, m}(x)^{t} \frac{2 \sqrt{1-x^{2}}}{\pi} d x=\delta_{n, k} I_{m+1} .
$$

If we denote

$$
\phi_{n, m}(z, y)=\Psi_{n, m}(z)\left[\begin{array}{c}
1 \\
y \\
\vdots \\
y^{m}
\end{array}\right],
$$

then to prove the orthogonality relation (3.5), it is enough to show that

$$
\int \phi_{1, m}(z, y) \phi_{1, m}(z, y)^{\dagger} d_{y} \mu=I_{m+1} \text {. }
$$

The proof of the last equality can be easily established by using two facts. The first one is to notice that

$$
\phi_{1,1}^{0}(z, y)=\frac{s_{1,1}^{2} z^{2}-2 s_{1,1} z y+1}{z \sqrt{1-s_{1,1}^{2}}},
$$

i.e. for the measure given in (3.3) we have

$$
d_{y} \mu=\frac{2}{\pi} \frac{\sqrt{1-y^{2}}}{\left|\phi_{1,1}^{0}(z, y)\right|^{2}} d y
$$

The second step is to connect $\phi_{1, m}(z, y)$ to the Chebyshev polynomials of the second kind. More precisely, we will show that

$$
\phi_{1, m}^{i}(z, y)= \begin{cases}\phi_{1,1}^{0}(z, y) U_{i}(y) & \text { if } i<m \\ \frac{1}{z} U_{m}(y)-s_{1,1} U_{m-1}(y) & \text { if } i=m .\end{cases}
$$

Notice that $1 / \phi_{1,1}^{0}(z, y)$ is essentially a generating function for the polynomials $U_{m}(y)$. This combined with elementary properties of the Chebyshev polynomials leads to

$$
\phi_{1, m}^{m}(z, y)=\sqrt{1-s_{1,1}^{2}} \phi_{1,1}^{0}(z, y) \sum_{j=0}^{\infty}\left(z s_{1,1}\right)^{j} U_{m+j}(y) .
$$

Equation (3.9) follows immediately from (3.10)-(3.12).

Thus it remains to establish (3.11). From the recurrence coefficients, it is clear that

$$
p_{0, m}^{j}(x, y)=\tilde{p}_{0, j}^{0}(x, y)=U_{j}(y) \text { for every } j \in \mathbb{N}_{0} .
$$

From (2.11) with $n=1$ it follows that

$$
p_{1, m}^{j}(x, y)=p_{1, m-1}^{j}(x, y) \text { for } 0 \leq j \leq m-2 .
$$

This implies that

$$
\phi_{1, m}^{j}(z, y)=\phi_{1, m-1}^{j}(z, y) \text { for } 0 \leq j \leq m-2 .
$$

Since $J_{n, m}^{3}=-\mathcal{K}_{n, m} \tilde{A}_{n-1, m}^{t}$ relation (2.12) for $n=1$ gives

$$
\begin{aligned}
& p_{1, m}^{j-1}+p_{1, m}^{j+1}=2 y p_{1, m-1}^{j}=2 y p_{1, m}^{j} \text { for } j \leq m-2 \\
& \sqrt{1-s_{1,1}^{2}} p_{1, m}^{m-2}+p_{1, m}^{m}=2 y p_{1, m-1}^{m-1}-s_{1,1} U_{m-1}(y),
\end{aligned}
$$


where in the first equality we used (3.14) and in the second (3.13). Using the definition of $\phi_{n, m}$, (3.13) and (3.16a) it follows that

$$
\phi_{1, m}^{j+1}+\phi_{1, m}^{j-1}=2 y \phi_{1, m}^{j} \text { for } j \leq m-2 .
$$

The last equation combined with the definition of $\phi_{1, m}$ leads by induction to $\phi_{1, m}^{j}=$ $\phi_{1,1}^{0} U_{j}(y)$ for $j \leq m-1$ thus proving the first part in equation (3.11).

Finally, beginning with the definition of $\phi_{1, m}^{m}$ and then using equation (3.16b) to eliminate $p_{1, m}^{m}$ yields

$$
\phi_{1, m}^{m}=2 y p_{1, m-1}^{m-1}-s_{1,1} U_{m-1}(y)-\sqrt{1-s_{1,1}^{2}} p_{1, m}^{m-2}-z U_{m}(y) .
$$

Now substituting in the definition for $\phi_{1, m-1}^{m-1}$, using the recurrence formula for $2 y U_{m-1}(y)$ and the definition for $\phi_{1, m}^{m-2}$ gives

$$
\phi_{1, m}^{m}=2 y \phi_{1, m-1}^{m-1}+z s_{1,1}^{2} U_{m-2}(y)-s_{1,1} U_{m-1}(y)-\sqrt{1-s_{1,1}^{2}} \phi_{1, m}^{m-2},
$$

from which it follows

$$
\phi_{1, m}^{m}-2 y \phi_{1, m-1}^{m-1}=\left(2 y s_{1,1}-\frac{1}{z}\right) U_{m-2}(y)-s_{1,1} U_{m-1}(y),
$$

which gives the second part in (3.11).

Remark 3.2. We have computed also the first few coefficients for the recurrence relations (1.1)-(1.2) in the total degree ordering. The computations suggest the following formulas

$$
\begin{gathered}
A_{x, n}=\frac{1}{2}\left[\begin{array}{lllll}
s_{1,1} & \sqrt{1-s_{1,1}^{2}} & & & \\
& & 1 & & \\
& & & \ddots & \\
& & & & 1
\end{array}\right] \\
A_{y, n}=\frac{1}{2}\left[\begin{array}{lllll}
1 & & & & \\
& 1 & & & \\
& & \ddots & & \\
& & & 1 & 0
\end{array}\right],
\end{gathered}
$$

and $B_{x, n}, B_{y, n}$ are $(n+1) \times(n+1)$ zero matrices.

\section{Two parameter Deformation of the Chebyshev polynomials}

Another deformation which leads to simple recurrence coefficients can be obtained by making the same choice of parameters as before except at levels $(1,0)$ and $(0,1)$. At level $(1,0)$ we leave $s_{1,0}$ free and we choose $s_{2,0}=\frac{1}{2}$. At level $(0,1)$ we put $s_{0,1}=s_{1,0} s_{1,1}$ and $s_{0,2}=\frac{1}{2}$. Using the same algorithm, we have this time two free parameters $s_{1,1}$ and $s_{1,0}$ and the recurrence coefficients take the form (we denote them by $A^{\prime}, B^{\prime}$, etc. in order to distinguish them from the one parameter 
deformation)

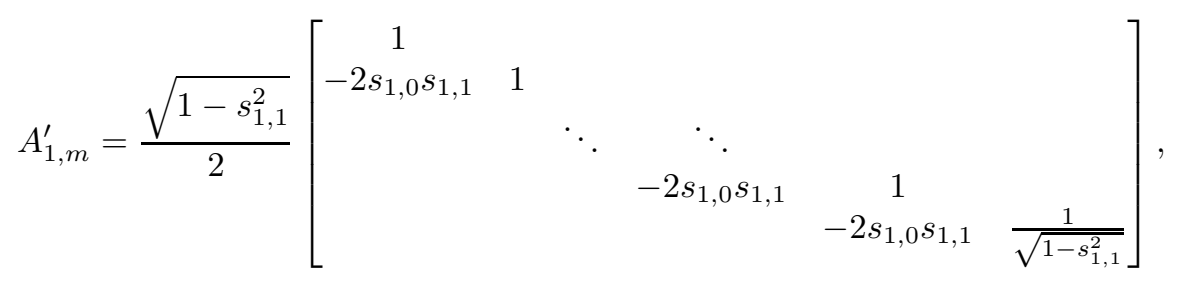

$A_{n, m}^{\prime}=\frac{1}{2} I_{m+1}$ for $n \geq 2$,

$$
\begin{gathered}
B_{0, m}^{\prime}=\frac{1}{2}\left[\begin{array}{ccccc}
2 s_{1,0} & s_{1,1} & & & \\
s_{1,1} & 2 s_{1,0}\left(1-s_{1,1}^{2}\right) & s_{1,1} & & \\
& & \ddots & & \\
& & s_{1,1} & 2 s_{1,0}\left(1-s_{1,1}^{2}\right) & s_{1,1} \\
& & & & \\
& & & & \\
B_{1, m}\left(1-s_{1,1}^{2}\right)
\end{array}\right], \\
B_{1,0}^{\prime}=\left[\begin{array}{lllll}
s_{1,0} s_{1,1}^{2} & & & & \\
& s_{1,0} s_{1,1}^{2} & & & \\
& & \ddots & & \\
& & & s_{1,0} s_{1,1}^{2} & 0
\end{array}\right], \quad B_{n, m}^{\prime}=0 \text { for } n \geq 2,
\end{gathered}
$$

and the coefficients $\mathcal{K}_{n, m}$ and $J_{n, m}^{1}$ are the same as in in the one parameter deformation.

Theorem 4.1. The polynomials $\mathbb{P}_{n, m}^{\prime}(x, y)$ corresponding to the parameters $s_{i, j}$ given by $s_{0,0}=1,\left|s_{1,0}\right|>\frac{1}{2}, s_{2,0}=\frac{1}{2}, s_{2 n-1,0}=0, s_{2 n, 0}=\frac{1}{2}$, for $n \geq 2$, $s_{0,1}=s_{1,0} s_{1,1}, s_{0,2}=\frac{1}{2}, s_{0,2 m-1}=0, s_{0,2 m}=\frac{1}{2}$ for $m \geq 2$, and

$$
s_{2 n-1,2 m-1}=s_{1,1}, \quad s_{2 n-1,2 m}=0, \quad s_{2 n, 2 m-1}=0, \quad s_{2 n, 2 m}=\frac{1}{2}, \quad n, m \in \mathbb{N}
$$

are orthonormal with respect to the measure

$$
\begin{aligned}
& d \mu^{\prime}(x, y)=\frac{2 z_{0}}{\pi^{2}\left(x_{0}-x\right)} \mu_{0}(x, y) \sqrt{1-x^{2}} \sqrt{1-y^{2}} d x d y \\
& \quad+\frac{2\left(1-z_{0}^{2}\right)}{\pi} \mu_{0}(x, y) \delta\left(x-x_{0}\right) \sqrt{1-y^{2}} d x d y,
\end{aligned}
$$

where $z_{0}=1 /\left(2 s_{1,0}\right)$ and

$$
\mu_{0}(x, y)=\frac{\left(1-s_{1,1}^{2}\right)}{4 s_{1,1}^{2}\left(x^{2}+y^{2}\right)-4 s_{1,1}\left(1+s_{1,1}^{2}\right) x y+\left(1-s_{1,1}^{2}\right)^{2}} .
$$

Proof. The shortest way to derive the orthogonality measure is to use Theorem 2.4 Indeed, let $\mathcal{L}_{m}$ be the second order difference operator corresponding to the second order difference equation (2.10) for the polynomials $\mathbb{P}_{n, m}(x, y)$ defined in Theorem 3.1] i.e. $\left(\mathcal{L}_{m}(f)\right)_{n}=A_{n+1, m} f_{n+1}+B_{n, m} f_{n}+A_{n, m}^{t} f_{n-1}$. Similarly, we denote by $\mathcal{L}_{m}^{\prime}$ the operator corresponding to the polynomials $\mathbb{P}_{n, m}^{\prime}(x, y)$ defined in Theorem 4.1. Then one can check that $\mathcal{L}_{m}$ and $\mathcal{L}_{m}^{\prime}$ are related by a Darboux 
transformation if we take $x_{0}=\frac{1}{2}\left(z_{0}+1 / z_{0}\right)=\left(4 s_{1,0}^{2}+1\right) /\left(4 s_{1,0}\right)$ and the operators $\mathcal{P}$ and $\mathcal{Q}$ are defined as follows

$$
\begin{array}{ll}
\mathcal{P}(f)_{n}=\frac{1}{2} f_{n+1}-\frac{1}{4 s_{1,0}} f_{n} \text { for } n \geq 1 & \text { and } \mathcal{P}(f)_{0}=A_{1, m} f_{1}-\frac{1}{4 s_{1,0}}\left(\alpha^{m}\right)^{t} f_{0} \\
\mathcal{Q}(f)_{n}=f_{n}-4 s_{1,0} A_{n, m}^{t} f_{n-1} \text { for } n \geq 1 & \text { and } \mathcal{Q}(f)_{0}=\alpha^{m} f_{0},
\end{array}
$$

where $\alpha^{m}$ is the $(m+1) \times(m+1)$ matrix given by

$$
\alpha^{m}=\left[\begin{array}{ccccc}
1 & & & & \\
-2 s_{1,0} s_{1,1} & 1 & & & \\
& -2 s_{1,0} s_{1,1} & 1 & & \\
& & \ddots & & \\
& & & -2 s_{1,0} s_{1,1} & 1
\end{array}\right] .
$$

From this relation it also follows that

$$
\hat{P}_{n, m}(x)=z_{0} P_{n, m}^{\prime}(x),
$$

where $\hat{P}_{n, m}$ are the polynomials defined in Theorem 2.4. Writing the orthogonality relations for $\hat{P}_{n, m}(x)$, using (4.3) and replacing $P_{n, m}^{\prime}(x)\left[1 y \ldots y^{m}\right]^{t}$ by $\mathbb{P}_{n, m}^{\prime}(x, y)$ we obtained the desired orthogonality.

Remark 4.2. Finally, we list the recurrence coefficients in the total degree ordering. The matrices $A_{x, n}$ and $A_{y, n}$ are the same as the matrices for the polynomials in Section 3, i.e. they are given by (3.17) and (3.18), respectively. For the $B_{x, n}$ we have

$$
B_{x .0}=\left[s_{1,0}\right], \quad B_{x, 1}=s_{1,0}\left[\begin{array}{cc}
1-s_{1,1}^{2} & -s_{1,1} \sqrt{1-s_{1,1}^{2}} \\
-s_{1,1} \sqrt{1-s_{1,1}^{2}} & s_{1,1}^{2}
\end{array}\right]
$$

and for $n \geq 2, B_{x, n}$ is the block matrix

$$
B_{x, n}=\left[\begin{array}{cc}
B_{x, 1} & 0 \\
0 & 0
\end{array}\right]
$$

The matrices $B_{y, n}$ are identically equal to zero for $n \geq 1$ and $B_{y, 0}=\left[s_{1,0} s_{1,1}\right]$.

\section{ACKNowledgments}

We would like to thank A. Delgado, F. A. Grünbaum and F. Marcellán for many useful discussions.

\section{REFERENCES}

[1] Ju. M. Berezanskii, Expansions in eigenfunctions of self-adjoint operators, Trans. Math. Mono. Amer. Math. Soc. 17 (1968).

[2] Ju. M. Berezanskii, Direct and inverse spectral problems for a Jacobi field Algebra i Analiz 9 (1997), 38-61; translation in St. Petersburg Math. J. (9), (1998), 1053-1071.

[3] A. Delgado, J. S. Geronimo, P. Iliev and F. Marcellán, Two variable orthogonal polynomials and structured matrices, SIAM J. Matr. Anal. Appl. 28 (2006), no. 1, 118-147.

[4] C. F. Dunkl, Intertwining operators and polynomials associated with the symmetric group Monatsh. Math. 126 (1998) 181-209.

[5] C. F. Dunkl and Y. Xu, Orthogonal polynomials of several variables, Encyclopedia of Mathematics and its Applications, 81. Cambridge University Press, Cambridge, 2001.

[6] A. J. Duran and F. A. Grünbaum, Structural formulas for orthogonal matrix polynomials satisfying second-order differential equations.I. Constr. Approx. 22, (2005),255-271 
[7] L. Fernández, T. E. Pérez and M. A. Piñar, Weak classical orthogonal polynomials in two variables, J. Comput. Appl. Math. 178 (2005), 191-203.

[8] M. I. Gekhtman and A. A. Kalyuzhny, On the orthogonal polynomials in several variables, Int. Equat. Oper. Theory 19 (1994) 404-418.

[9] J. S. Geronimo, Scattering theory and matrix orthogonal polynomials on the real line, Circuits Systems Signal Process 1 (1982), 471-495.

[10] J. S. Geronimo and H. J. Woerdeman, Positive extensions, Fejér-Riesz factorization and autoregressive filters in two variables, Ann. of Math. (2) 160 (2004), 839-906.

[11] J. S. Geronimo and H. J. Woerdeman, Two variable orthogonal polynomials on the bi-circle and structured matrices.

[12] D. Jackson, Formal properties of orthogonal polynomials in two variables, Duke Math. J. 2 (1936) 423-434.

[13] Y. J. Kim, K. H. Kwon and J. K. Lee, Multi-variate orthogonal polynomials and second order partial differential equations, Commun. Appl. Anal. 6 (2002) 479-504.

[14] T. H. Koornwinder, Orthogonal polynomials in two variables which are eigenfunctions of two algebraically independent partial differential operators, I, II. Indag. Math. 36 (1974) 48-66.

[15] T. H. Koornwinder, Two variable analogues of the classical orthogonal polynomials, Theory and Applications of Special Functions, ed R. Askey Academic Press, N.Y. (1975) 435-495.

[16] T. H. Koornwinder, Askey-Wilson polynomials for root systems of type BC, in Hypergeometric functions on domains of positivity, Jack polynomials, and applications, Contemp. Math. 138 Amer. Math. Soc. (1992) 189-204.

[17] M. A. Kowalski, The recursion formulas for orthogonal polynomials in $n$ variables, SIAM J. Math. Anal. 13 (1982), 309-315.

[18] M. A. Kowalski, Orthogonality and recursion formulas for polynomials in $n$ variables, SIAM J. Math. Anal. 13 (1982), 316-323.

[19] H. L. Krall and I. M. Sheffer, Orthogonal polynomials in two variables, Ann. Mat. Pura ed Appl. 76 (4) (1967) 325-376.

[20] M. G. Krein, Infinite J-matrices and the matrix moment problem, Dokl. Akad. Nauk. SSSR. 69 (1949) 125-128.

[21] K. H. Kwon, J. K. Lee and L. L. Littlejohn, Orthogonal polynomial eigenfunctions of second order partial differential equations, Trans. Amer. Math. Soc. 353 (2001) 3629-3647.

[22] I. G. Macdonald, Symmetric functions and Hall polynomials, Oxford University Press, New York, 1995.

[23] F. Marcellán and H. O. Yakhlef, Recent trends on analytic properties of matrix orthogonal polynomials, approximation theory, and harmonic analysis (Inzel, 2000), Electron. Trans. Numer. Anal. 14, (2002), 127-141

[24] V. P. Serebrjakov, The inverse problem of scattering theory for difference equations with matrix coefficients. Soviet Math. Dokl. 21, (1980), 148.

[25] P. K. Suetin, Orthogonal polynomials in two variables, Analytical Methods and Special Functions, 3, Gordon and Breach Science Publishers, Amsterdam, 1999.

[26] A. Sinap and W. Van Assche Orthogonal matrix polynomials and applications Proc. sixth International Cong. on Comp. and Applied Math. J. Comput. Appl. Math. 66, (1996) 27-52.

[27] Y. Xu, On multivariate orthogonal polynomials, SIAM J. Math. Anal. 24 (1993) 783-794.

School of Mathematics, Georgia Institute of Technology, Atlanta, Ga 30332-0160, USA

E-mail address: geronimo@math.gatech.edu

School of Mathematics, Georgia Institute of Technology, Atlanta, Ga 30332-0160, USA

E-mail address: iliev@math.gatech.edu 